\title{
On the Mechanism of Hydrogen Storage in a Metal-Organic Framework (MOF) Material
}

\author{
Jonathan L. Belof, Abraham C. Stern, Mohamed Eddaoudi and Brian Space* \\ Department of Chemistry \\ University of South Florida \\ 4202 E. Fowler Ave., CHE205 \\ Tampa, FL 33620-5250 \\ *space@cas.usf.edu
}

\section{Supporting Information - Hybrid Monte Carlo}

While MD simulations with many-body polarizable forces can yield insight into the behavior of complex systems, they are also reputed for their instability and complexity of implementation; development of stable and efficient polarizable dynamics is currently an active area of research in computational chemistry and physics. ${ }^{1-4}$ The polarizable forces are troublesome and expensive to calculate; the polarization potential energy is (relatively) less problematic and is easier to compute via the dipole field equations.

Our initial approach was to perform a hybrid Monte Carlo $^{5}$ (HMC) calculation whereby the potential energy, especially including the polarization energy, was used in the Metropolis acceptance function but the system configuration was globally propagated by MD using a nonpolarizable force field. In this way, we would circumvent the need to compute polarizable forces and yet properly sample the configuration space that would reflect polarization effects - all the while, getting the benefit of short correlation times and efficient sampling of phase space due to the global moves being made (as opposed to the long correlation times that are typical of many-body potentials in the standard Monte Carlo scheme).

Unfortunately, the above HMC approach proved unworkable for a number of reasons. While the HMC method has been demonstrated to work well for LennardJones fluids ${ }^{6}$ and fermionic particles, ${ }^{5}$ in a system with many degrees of freedom we found a difficulty in the standard momentum resampling scheme that is not, to our knowledge, specifically addressed in the literature. It was our experience that completely resampling the momenta hindered the molecular rotations of the system, even in the non-polarizable scenario, and the structure for bulk molecular fluids that we tested (such as hydrogen and water) did not accurately correspond to the known structure, as was evident by the comparison of radial distri- bution functions from MD and Monte Carlo. However, for a Lennard-Jones system (i.e. possessing only translational degrees of freedom) the complete momenta resampling scheme yields the correct structure. It has been clarified that a complete resampling of momenta from a Boltzmann distribution is not required, ${ }^{7}$ and in fact we implemented an alternative momentum update recently given in the literature ${ }^{8}$ whereby the momentum vectors can be less drastically perturbed and then a Metropolis evaluation of the kinetic energy is performed:

$$
\begin{array}{r}
\{\overrightarrow{p \prime}\}=\{\vec{p}\}+\alpha\{\vec{\pi}\} \\
\min \left\{1, \exp \left[-\beta\left(\frac{p^{\prime}}{2 m}-\frac{p^{2}}{2 m}\right)\right]\right\}
\end{array}
$$

where $\{\vec{p}\}$ is the set of momentum vectors in the system, $\{\vec{\pi}\}$ is a set of vectors randomly sampled from a Boltzmann distribution and $\alpha$ is a free parameter used to tune the acceptance ratio. This update scheme restored the correct rotational dynamics and structure for the bulk systems studied with HMC. While the prime motivation for the initial development of alternative momentum resampling schemes has been to dispensably improve the efficiency, it is our view that less drastic momentum resampling is requisite in simulating systems with rotational degrees of freedom. However, a second (unfortunately insurmountable) difficulty with utilizing HMC to study soc-MOF was in using a polarizable MC potential with non-polarizable dynamics: in situations where the polarization effects are significant (e.g. soc-MOF at low temperature), the polarizable and non-polarizable potential energy surfaces are simply discrepant enough to prevent the adequate sampling of phase space (this again stresses the importance of polarization interactions for $\mathrm{H}_{2}$ in socMOF). Thus, the computationally demanding standard Monte Carlo scheme was implemented.
1 D. N. Bernardo, Y. Ding, K. Krogh-Jespersen, and R. M. Levy, J. Phys. Chem. 98, 4180 (1994).

2 S. S. X. Christian J. Burnham, Jichen Li and M. Leslie, J. Chem. Phys. 110, 4566 (1999).
${ }^{3}$ P. Ren and J. W. Ponder, J. Phys. Chem. B 107, 5933 (2003).

${ }^{4}$ M. Souaille and J. C. Smith, Mol. Phys. 87, 1333 (1996).

5 S. Duane, A. Kennedy, B. J. Pendleton, and D. Roweth, 
Phys. Lett. B 195, 216 (1987).

${ }^{6}$ B. Mehlig, D. Heermann, and B. Forrest, Phys. Rev. B 45, 679 (1992).

7 A. M. Horowitz, Phys. Lett. B 268, 247 (1991).
8 E. Akhmatskaya and S. Reich, Lecture Notes in Computational Science and Engineering 49, 141 (2005). 